\section{Dor nas costas em população adulta: estudo de base populacional em Campinas, São Paulo, Brasil}

\author{
Back pain in adults: a population-based study in \\ Campinas, São Paulo State, Brazil
}

El dolor de espalda en la población adulta: estudio de base poblacional en Campinas, São Paulo, Brasil
Aparecida Mari Iguti 1

Tássia Fraga Bastos 1

Marilisa Berti de Azevedo Barros 1

\footnotetext{
1 Faculdade de Ciências Médicas, Universidade Estadual de Campinas, Campinas, Brasil.

Correspondência A. M. Iguti

Departamento de Saúde Coletiva, Faculdade de Ciências Médicas, Universidade Estadual de Campinas.

Rua Tessalia Vieira de Camargo 126, Campinas, SP 13083-971, Brasil. iguti@fcm.unicamp.br
}

\begin{abstract}
This study aimed to estimate the prevalence of back pain in the urban population of Campinas, São Paulo State, Brazil, and identify the most affected groups. Data were obtained from a population survey in 2008-2009 that included 1,118 individuals aged 18-59 years. Overall prevalence of back pain was $30.6 \%$ (34.4\% in women and $26.5 \%$ in men). After adjustment, prevalence was higher among women, those with less schooling, people working more than 40 hours a week, smokers and former smokers, those with no leisure-time physical activity, and those who were physically active in household chores and at work. Prevalence increased with age and with the number of children, number of diseases and health problems, and those with worse self-rated health. The results confirm the high prevalence of low back pain and the associated incapacity, indicating the population groups that require heightened attention from health services.
\end{abstract}

Back Pain; Adult Health; Health Surveys

\section{Resumo}

Este estudo teve por objetivos estimar a prevalência de dor nas costas na população urbana de Campinas, São Paulo, Brasil, e identificar os subgrupos populacionais mais afetados pelo problema. Os dados foram obtidos na pesquisa populacional realizada em 2008 e 2009, que envolveu 1.118 indivíduos com idades de 18 a 59 anos. A prevalência de dor nas costas foi de $30,6 \%$, acometendo 34,4\% das mulheres e 26,5\% dos homens. Após ajustes, a prevalência se mostrou mais elevada nas mulheres, nos de menor escolaridade, nos que trabalhavam mais de 40 horas por semana, nos fumantes e ex-fumantes, nos que não faziam atividade física em contexto de lazer e nos ativos nas atividades domésticas e de trabalho. A prevalência revelou-se crescente com a idade e com o número de filhos, com o número de morbidades e problemas de saúde e com a piora da autoavaliação da saúde. Os resultados confirmam a elevada prevalência do problema e das limitações que provoca, indicando os segmentos sociodemográficos que merecem maior atenção em ações de prevenção e controle dos serviços de saúde.

Dor nas Costas; Saúde do Adulto; Inquéritos Epidemiológicos 


\section{Introdução}

Dor nas costas é um termo coloquial utilizado para designar as algias relacionadas à coluna vertebral. Embora a maioria se refira às lombalgias, os relatos também incluem as dorsalgias e cervicalgias. Trata-se de um problema amplamente difundido e relevante pela prevalência elevada, pela demanda que acarreta aos serviços de assistência médica e pelas limitações que provoca com consequente prejuízo à qualidade de vida dos pacientes.

Uma revisão sistemática que investigou a prevalência global de dor nas costas por meio de estudos transversais constatou que, considerando a média de diversas porcentagens, $31 \%$ da população adulta das diferentes localidades estudadas são acometidas por este problema de saúde 1. No entanto, os autores salientam que essa prevalência deve ser vista com cautela, por conta da heterogeneidade metodológica entre os estudos analisados. No Brasil, dados da Pesquisa Nacional por Amostra de Domicílios (PNAD) apontaram que em 2008 a doença nas costas/ coluna foi a segunda morbidade mais prevalente entre as investigadas, atingindo $30 \%$ dos brasileiros na faixa de 50 a 59 anos de idade 2 .

As pesquisas destacam o elevado porcentual da população que demanda serviço de saúde por problemas de lombalgia 1 e a elevada frequência de limitação das atividades diárias entre os indivíduos afetados, incluindo afastamento do trabalho, aposentadoria por invalidez e dias com restrição ao leito 3,4,5

Entre 291 condições de saúde avaliadas em estudo sobre a carga global das doenças, a dor lombar foi a que mais contribuiu para incapacidades, medidas pelo indicador de anos vividos com incapacidades, e ocupou a sexta posição em termos de carga global, avaliada pelos anos de vida perdidos ajustados por incapacidades, que estimam os anos de vida perdidos seja por morte prematura ou por incapacidades em relação a uma esperança de vida ideal 6 .

Uma parcela significativa das pesquisas sobre as algias lombares é orientada aos grupos ocupacionais, por se tratar de evento com impactos sobre o absenteísmo, custos à produtividade e demandas previdenciárias. Entretanto, vários estudos relacionados à população geral têm sido realizados para estimar a prevalência do problema e verificar as associações existentes entre a presença de eventos álgicos e diferentes modalidades de fatores. Ressalte-se que poucos estudos populacionais brasileiros têm sido conduzidos sobre essa temática $2,7,8,9,10$ e que a prevalência de dores nas costas entre brasileiros permanece pouco conhecida.
Este trabalho teve por objetivos estimar a prevalência de dores nas costas na população residente na região urbana do Município de Campinas, São Paulo, Brasil, e identificar os subgrupos populacionais mais afetados pelo problema.

\section{Método}

Este é um estudo transversal, de base populacional, que envolveu 1.118 indivíduos, com 18 a 59 anos de idade, não institucionalizados, residentes na área urbana do Município de Campinas. Os dados são originários do Inquérito de Saúde de Campinas (ISACamp 2008), realizado em 2008 e 2009, e foram obtidos por meio de questionários estruturados, aplicados por entrevistadores treinados e supervisionados.

Para a seleção da amostra do ISACamp 2008, utilizou-se procedimentos de amostragem probabilística, por conglomerados e em dois estágios. No primeiro, foram sorteados cinquenta setores censitários da área urbana do Município de Campinas, com probabilidade proporcional ao tamanho, correspondente ao número de domicílios. No segundo estágio, dos setores amostrados foram sorteados domicílios de forma a garantir um tamanho mínimo de amostra. O número de domicílios sorteados foi estimado para que fossem encontradas 1.000 pessoas em cada domínio de idade (adolescentes, adultos e idosos). O número de domicílios foi calculado com base na média esperada de indivíduos, de cada domínio de idade, nas residências da área urbana do Município de Campinas. Atendendo à proposta do inquérito, de estudar eventos relacionados à saúde de três subgrupos da população, adolescentes (10 a 19 anos), adultos (20 a 59) e idosos (60 anos ou mais), foram tomadas amostras independentes de domicílios para cada um destes domínios.

O tamanho da amostra foi obtido considerando-se a situação correspondente à máxima variabilidade para a frequência dos eventos estudados (valor de $\mathrm{p}=0,50$ ), um coeficiente de 95\% de confiança na determinação dos intervalos de confiança $(z=1,96)$, erro de amostragem entre 4 e 5 pontos percentuais e efeito de delineamento de 2, totalizando 1.000 indivíduos para cada domínio de idade. Esperando-se uma taxa de $80 \%$ de cobertura e resposta, o tamanho da amostra foi corrigido para 1.250. Para atingir esse número para cada domínio, após atualização em campo da listagem de endereços dos setores sorteados, foram amostrados 2.150, 700 e 3.900 domicílios para as entrevistas com adolescentes, adultos e idosos, respectivamente. O número de domicílios foi calculado com base na média esperada de pessoas, de cada domínio de idade, 
nas residências da área urbana do Município de Campinas, baseando-se nos dados do censo demográfico. Em cada domicílio sorteado foram entrevistados todos os moradores da faixa etária selecionada 11 .

A variável dependente analisada neste trabalho foi a presença de dor nas costas/problema na coluna relatada pelos entrevistados. Essa informação foi obtida por meio de um checklist que incluiu vários problemas de saúde e deficiências, entre os quais a dor nas costas, que seguiam a pergunta: "Você tem algum dos seguintes problemas de saúde e/ou deficiências?" Esse checklist vinha após um outro em que eram feitas questões relativas à presença de doenças crônicas diagnosticadas por médico. O checklist de problemas de saúde incluía condições que são recorrentes e era encabeçado por "dor de cabeça frequente/ enxaqueca”. Assim, não era perguntado se a pessoa estava sentindo dor nas costas no momento da entrevista e sim se tinha o problema. Também não foi especificado o período de prevalência da dor, incluindo-se então na análise deste trabalho todas as pessoas que relataram ter dor nas costas ou problema na coluna, independentemente se sentia no momento da entrevista ou se era uma queixa recorrente do entrevistado.

Foram também analisados os seguintes conjuntos de variáveis independentes:

a) Demográficas e socioeconômicas: sexo; faixa etária; situação conjugal; número de filhos; escolaridade (em anos de estudos); renda familiar per capita (em salários mínimos); ocupação; carga horária semanal no trabalho; cobertura por plano de saúde.

b) Comportamentos relacionados à saúde: uso abusivo de bebida alcoólica (mensurado por meio do Alcohol Use Disorders Identification Test - AUDIT, com 8 ou mais pontos sendo considerado positivo) 12; tabagismo (fumante, ex-fumante, nunca fumou); atividade física nos domínios de lazer, doméstico, trabalho e deslocamento, classificados em: ativo (pelo menos 150 minutos por semana, por pelo menos 3 dias na semana), insuficientemente ativo (menos de 150 minutos por semana ou por menos de 3 dias na semana), inativo (nenhum tempo dedicado à atividade física de lazer na semana); horas diárias de sono; horas diárias no computador; e horas diárias assistindo à televisão.

c) Condições de saúde e morbidades: doenças crônicas obtidas com base em um checklist que incluía, entre outras: hipertensão arterial, diabetes e artrite/reumatismo/artrose; número total de doenças crônicas; problemas de saúde obtidos em checklist que incluía, entre outros: dor de cabeça/enxaqueca, tendinite/LER/DORT e insônia; número total de problemas de saúde re- feridos (excluindo dor nas costas/problemas na coluna); índice de massa corporal (IMC), usando-se peso e altura referidos e as categorias estabelecidas pela Organização Mundial da Saúde 13 para adultos: baixo peso e eutrófico (IMC $<25 \mathrm{~kg}$ / $\left.\mathrm{m}^{2}\right)$, sobrepeso $\left(25-29 \mathrm{~kg} / \mathrm{m}^{2}\right)$ e obeso $(\geq 30 \mathrm{~kg} /$ $\mathrm{m}^{2}$ ); transtorno mental comum (TMC), avaliado pelo Self Reporting Questionnaire (SRQ-20) utilizando-se ponto de corte 6 para homens e 8 para mulheres ${ }^{14}$ e autoavaliação da saúde.

A associação entre as variáveis foi verificada pelo teste qui-quadrado com nível de significância de 5\%. Modelos de regressão simples e múltipla de Poisson com variância robusta foram utilizados para estimar as razões de prevalência brutas e ajustadas e os respectivos intervalos de 95\% de confiança (IC95\%). O modelo final de regressão múltipla de Poisson foi desenvolvido em quatro etapas, com a entrada sucessiva de quatro conjuntos de variáveis: primeira etapa: variáveis demográficas e socioeconômicas; segunda: comportamentos relacionados à saúde; terceira: condições de saúde e morbidades; quarta etapa: autoavaliação de saúde. Em cada uma delas foram incluídas as variáveis daquele conjunto que apresentaram na análise bivariada associação com valor de $\mathrm{p}$ inferior a 0,20 , permanecendo ao final de cada etapa as que alcançaram nível de significância de $5 \%$ (valor de $p<0,05$ ). As variáveis de uma etapa eram ajustadas pelas variáveis que haviam permanecido como significativas nas etapas prévias, além de ajustadas pelas variáveis de seu próprio conjunto. As variáveis IMC e transtorno mental comum foram incluídas na terceira etapa do modelo, obedecendo ao critério estabelecido, conforme descrito na seção Método. No entanto, ao serem introduzidas no modelo e sofrendo ajuste pelas variáveis que persistiram das primeiras e segundas etapas e das variáveis que foram sendo incluídas da terceira etapa, perderam significância, não permanecendo assim no modelo final.

Em todas as análises considerou-se o efeito do desenho amostral e as ponderações para análise de inquéritos baseados em delineamentos complexos do programa Stata 11.0 (StataCorp LP, College Station, Estados Unidos).

$\mathrm{O}$ projeto de pesquisa foi aprovado pela Comissão de Ética da Universidade Estadual de Campinas (parecer no 079/2007).

\section{Resultados}

A população de estudo foi composta por 1.118 pessoas com idades entre 18 e 59 anos (526 homens e 592 mulheres). A prevalência de dor nas costas/problema na coluna foi de $30,6 \%$ (IC95\%: 
26,6-35,0), acometendo 34,4\% (IC95\%: 29,0-40,3) das mulheres e 26,5\% (IC95\%: 22,4-31,1) dos homens. Dentre os que relataram ter dor nas costas, 21,4\% (IC95\%: 14,4-30,5) afirmaram que esta condição limitava suas atividades diárias.

A Tabela 1 apresenta a prevalência de dor nas costas segundo variáveis demográficas e socioeconômicas. Após ajuste por idade e sexo, as razões de prevalência revelam-se crescentes com o aumento da idade e do número de filhos, e significativamente mais elevada no sexo feminino, no segmento de menor escolaridade e entre as pessoas que trabalhavam mais de 40 horas por semana, já a variável renda familiar per capita não mostrou associação com dor nas costas nem na análise bruta. Quanto à condição de inserção em atividade ocupacional, a categoria de estudantes apresentou prevalência inferior às dos demais segmentos. A associação com o número de filhos foi constatada tanto para as mulheres $(R P=2,20$; IC95\%: 1,42-3,41) quanto para os homens (RP = 2,29; IC95\%: 1,22-4,31) (dados não apresentados em tabela). A variável renda familiar per capita não foi associada à dor nas costas.

Quanto às variáveis de comportamentos relacionados à saúde (Tabela 2), uso abusivo de álcool, hábito de fumar e inatividade física no lazer revelaram-se positivamente associados à prevalência de dor nas costas, mesmo após o ajuste por idade e sexo. Pessoas que praticavam atividade física doméstica, de trabalho e de deslocamento apresentaram maiores prevalências de dor nas costas.

Analisando-se a prevalência de dor na coluna, segundo morbidades, problemas de saúde e autoavaliação da saúde (Tabela 3), nota-se que após ajuste por idade e sexo, entre as variáveis apresentadas, apenas diabetes e tendinite/LER/ DORT não estiveram associadas às algias lombares. Apresentaram maior prevalência de dores nas costas os indivíduos com artrite/artrose, dor de cabeça, os hipertensos, os que referiram ter insônia, os que apresentaram transtorno mental comum e os obesos. Maiores magnitudes de associação com dor nas costas foram evidenciadas entre os que avaliaram a própria saúde como ruim ou muito ruim $(R P=2,93)$ e entre aqueles que relataram três ou mais problemas de saúde $(\mathrm{RP}=2,97)$.

Na Tabela 4 são apresentadas as variáveis que permaneceram associadas à dor nas costas, após os ajustes em cada etapa do modelo. Dentre os segmentos demográficos e socioeconômicos, registraram maiores prevalências: sexo feminino, idades de 50 a 59 anos, indivíduos com carga semanal de trabalho maior que 40 horas e maior número de filhos. Os tabagistas e os que não praticavam atividade física em contexto de lazer apresentaram associação positiva com dor nas costas, e os que eram ativos no contexto doméstico e de trabalho registraram maiores prevalências. Aqueles que relataram assistir à televisão por quatro horas ou mais no dia apresentaram probabilidade $35 \%$ maior de ter dor nas costas. Ter um ou mais problemas de saúde manteve associação estatisticamente significativa com a variável de desfecho, assim como a autoavaliação de saúde boa e ruim/muito ruim.

\section{Discussão}

Neste trabalho, cerca de um terço da população adulta de Campinas $(30,6 \%)$ relatou dor nas costas, uma prevalência elevada que está situada na ampla faixa das observadas por outros estudos 1 e que é praticamente similar à prevalência média geral estimada em revisão sistemática desenvolvida sobre o tema. No Brasil, dados da PNAD revelaram prevalências de doenças de coluna ou das costas diagnosticadas por médico de $8 \%$ na faixa de 20 a 29 anos e de $30 \%$ na de 50 a 592 . Grande diversidade de prevalências tem sido verificada também entre as pesquisas desenvolvidas no Brasil. Um estudo realizado em Pelotas, Rio Grande do Sul, encontrou prevalência de dor lombar crônica de 4,2\% e duração média da dor de 82,6 dias 7,8. Outro trabalho no mesmo município apresentou a prevalência de dor nas costas de $63,1 \%$. Em um estudo transversal conduzido na cidade de Salvador, Bahia, observou-se a prevalência de dor lombar crônica de $14,7 \%$ 10. As diferentes prevalências observadas entre os estudos decorrem em grande parte dos diferentes métodos e definições utilizados, de especificação ou não da localização da dor e da condição de ser ou não crônica, das faixas de idades estudadas, do instrumento e do meio utilizado de obtenção dos dados 1 .

A maior prevalência de dores nas costas em mulheres (34,4\% versus $26,5 \%$ nos homens) encontrada na presente pesquisa também foi constatada na maioria dos estudos realizados em diferentes países $1,2,3,5,15,16,17,18,19,20$, e tem sido atribuída por vários autores a fatores hormonais e reprodutivos. Em um estudo específico com mulheres encontrou-se associação das lombalgias crônicas com gravidez anterior, gravidez em idade precoce, duração do uso de contraceptivos orais, menstruação irregular e prolongada, histerectomia e reposição hormonal com estrógenos na menopausa ${ }^{20}$. Autores têm apontado a maior prevalência de osteoporose nas mulheres, e as diferenças socioculturais relacionadas à expressão dos sintomas álgicos que também devem ser consideradas 15 . 
Tabela 1

Prevalência de dor nas costas segundo variáveis demográficas e socioeconômicas na população adulta (18 a 59 anos) de Campinas, São Paulo, Brasil, em 2008. Inquérito de Saúde de Campinas (ISACamp), 2008/2009.

\begin{tabular}{|c|c|c|c|c|}
\hline Variáveis & $\mathrm{n}$ & Prevalência (\%) & RP bruta (IC95\%) & RP ajustada * (IC95\%) \\
\hline Sexo & & $p=0,0062 \star \star$ & & \\
\hline Masculino & 526 & 26,5 & 1,00 & 1,00 \\
\hline Feminino & 592 & 34,4 & $1,30(1,08-1,56)$ & $1,27(1,07-1,52)$ \\
\hline Total & 1.118 & 30,6 & & \\
\hline Faixa etária (anos) & & $p<0,0001$ ** & & \\
\hline $18-29$ & 466 & 20,3 & 1,00 & 1,00 \\
\hline $30-39$ & 232 & 30,5 & $1,50(1,16-1,95)$ & $1,48(1,13-1,92)$ \\
\hline $40-49$ & 224 & 35,4 & $1,74(1,34-2,26)$ & $1,71(1,32-2,22)$ \\
\hline $50-59$ & 196 & 44,0 & $2,17(1,69-2,77)$ & $2,16(1,68-2,77)$ \\
\hline Situação conjugal & & $p=0,0002$ ** & & \\
\hline Casado & 606 & 33,7 & $1,59(1,25-2,01)$ & $1,22(0,96-1,55)$ \\
\hline Divorciado & 91 & 40,4 & $1,91(1,33-2,73)$ & $1,31(0,92-1,86)$ \\
\hline Solteiro & 406 & 21,2 & 1,00 & 1 \\
\hline Viúvo & 15 & 39,9 & $1,88(1,00-3,53)$ & $1,08(0,60-1,94)$ \\
\hline Número de filhos & & $p<0,0001$ ** & & \\
\hline 0 & 439 & 18,6 & 1,00 & 1,00 \\
\hline $1-2$ & 469 & 30,4 & $1,64(1,25-2,14)$ & $1,44(1,09-1,91)$ \\
\hline$\geq 3$ & 210 & 51,4 & $2,77(2,04-3,77)$ & $2,22(1,53-3,24)$ \\
\hline Escolaridade (anos) & & $p=0,0004 \star \star$ & & \\
\hline $0-8$ & 410 & 39,8 & $1,71(1,23-2,36)$ & $1,52(1,09-2,12)$ \\
\hline $9-11$ & 393 & 26,5 & $1,14(0,83-1,56)$ & $1,23(0,89-1,70)$ \\
\hline 12 ou mais & 315 & 23,3 & 1,00 & 1,00 \\
\hline Renda familiar per capita (salários mínimos) & & $p=0,8527 * \star$ & & \\
\hline Até 0,5 & 193 & 31,4 & $1,14(0,76-1,70)$ & $1,15(0,75-1,77)$ \\
\hline$>0,5-1$ & 293 & 31,8 & $1,15(0,84-1,59)$ & $1,26(0,91-1,73)$ \\
\hline$>1-2$ & 297 & 32,2 & $1,17(0.86-1,58)$ & $1,24(0,92-1,67)$ \\
\hline$>2-3$ & 134 & 28,4 & $1,03(0,70-1,51)$ & $1,04(0,70-1,56)$ \\
\hline$>3$ ou mais & 201 & 27,6 & 1,00 & 1,00 \\
\hline Ocupação & & $p=0,0004$ ** & & \\
\hline Em atividade & 775 & 30,2 & 1,00 & 1,00 \\
\hline Desempregado & 72 & 18,5 & $0,61(0,35-1,08)$ & $0,68(0,39-1,18)$ \\
\hline Aposentado & 41 & 39,3 & $1,30(0,82-2,08)$ & $0,96(0,58-1,58)$ \\
\hline Dona de casa & 149 & 42,7 & $1,44(1,17-1,77)$ & $1,18(0,92-1,51)$ \\
\hline Estudante & 70 & 10,1 & $0,35(0,18-0,68)$ & $0,47(0,23-0,97)$ \\
\hline Carga horária semanal de trabalho (horas) & & $p=0,1517 \star \star$ & & \\
\hline Não trabalha & 332 & 32,3 & $1,37(1,07-1,75)$ & $1,17(0,90-1,53)$ \\
\hline$<40$ & 210 & 30,1 & $1,27(0,91-1,79)$ & $1,09(0,78-1,53)$ \\
\hline 40 & 228 & 23,6 & 1,00 & 1,00 \\
\hline$>40$ & 323 & 32,7 & $1,39(1,00-1,92)$ & $1,40(1,02-1,92)$ \\
\hline Plano de saúde & & $p=0,2352 \star \star *$ & & \\
\hline Sim & 476 & 28,6 & 1,00 & 1,00 \\
\hline Não & 641 & 32,2 & $1,12(0,92-1,37)$ & $1,14(0,93-1,40)$ \\
\hline
\end{tabular}

IC95\%: intervalo de 95\% de confiança; RP: razão de prevalência.

* Ajustada por idade e/ou sexo;

* Valor de $p$ do teste qui-quadrado. 
Prevalência de dor nas costas segundo variáveis de comportamentos relacionadas à saúde na população adulta (18 a 59 anos) de Campinas, São Paulo, Brasil em 2008. Inquérito de Saúde de Campinas (ISACamp), 2008/2009.

\begin{tabular}{|c|c|c|c|c|}
\hline Comportamentos & $\mathbf{n}$ & Prevalência (\%) & RP bruta (IC95\%) & RP ajustada * (IC95\%) \\
\hline AUDIT & & $p=0,0833 * *$ & & \\
\hline Positivo & 101 & 38,5 & $1,30(0,98-1,72)$ & $1,43(1,05-1,93)$ \\
\hline Negativo & 1.016 & 29,7 & 1,00 & 1,00 \\
\hline Tabagismo & & $p=0,0017 * *$ & & \\
\hline Fumante & 211 & 37,1 & $1,40(1,11-1,76)$ & $1,36(1,08-1,70)$ \\
\hline Ex-fumante & 116 & 43,7 & $1,65(1,20-2,26)$ & $1,49(1,09-2,04)$ \\
\hline Nunca fumou & 789 & 26,5 & 1,00 & 1,00 \\
\hline Atividade física no lazer & & $p=0,0015^{\star *}$ & & \\
\hline Ativo & 226 & 20,6 & 1,00 & 1,00 \\
\hline Insuficientemente ativo & 165 & 25,2 & $1,22(0,77-1,95)$ & $1,32(0,85-2,08)$ \\
\hline Inativo & 727 & 34,8 & $1,69(1,27-2,25)$ & $1,62(1,24-2,12)$ \\
\hline Atividade física doméstica & & $p=0,0028$ & & \\
\hline Ativo & 183 & 43,9 & 1,00 & 1,00 \\
\hline Insuficientemente ativo & 172 & 27,8 & $0,63(0,45-0,89)$ & $0,69(0,50-0,94)$ \\
\hline Inativo & 763 & 28,0 & $0,64(0,49-0,83)$ & $0,73(0,56-0,95)$ \\
\hline Atividade física no trabalho & & $p=0,0044$ & & \\
\hline Ativo & 335 & 35,6 & 1,00 & 1,00 \\
\hline Insuficientemente ativo & 37 & 47,5 & $1,33(0,93-1,91)$ & $1,24(0,87-1,79)$ \\
\hline Inativo & 746 & 27,4 & $0,77(0,63-0,94)$ & $0,71(0,56-0,89)$ \\
\hline Atividade física de deslocamento & & $p=0,0342$ & & \\
\hline Ativo & 366 & 36,1 & 1,00 & 1,00 \\
\hline Insuficientemente ativo & 299 & 29,0 & $0,81(0,63-1,02)$ & $0,76(0,59-0.98)$ \\
\hline Inativo & 453 & 27,4 & $0,76(0,61-0,95)$ & $0,71(0,56-0,88)$ \\
\hline Horas de sono & & $p=0587 \star \star$ & & \\
\hline Até 6 & 161 & 41,2 & $1,40(1,05-1,87)$ & $1,29(0,97-1,71)$ \\
\hline $7-8$ & 694 & 29,4 & 1,00 & 1,00 \\
\hline 9 ou mais & 261 & 27,1 & $0,92(0,65-1,32)$ & $0,99(0,69-1,41)$ \\
\hline Horas assistindo televisão & & $p=0,0283 * \star$ & & \\
\hline Até 1 & 276 & 27,6 & 1,00 & 1,00 \\
\hline 2 & 302 & 25,6 & $0,92(0,71-1,20)$ & $0,91(0,70-1,19)$ \\
\hline 3 & 274 & 32,6 & $1,18(0,92-1,51)$ & $1,16(0,90-1,50)$ \\
\hline 4 ou mais & 261 & 38,3 & $1,39(1,01-1,90)$ & $1,24(0,94-1,79)$ \\
\hline
\end{tabular}

AUDIT: Alcohol Use Disorders Identification Test; IC95\%: intervalo de 95\% de confiança; RP: razão de prevalência.

* Ajustada por idade e sexo;

** Valor de $\mathrm{p}$ do teste qui-quadrado.

O aumento da prevalência de dores nas costas com o avanço da idade é achado frequente na literatura 1,2,6,15,21,22,23, incluindo o aumento da prevalência de lombalgia com incapacidade 15,24. Alguns estudos têm detectado aumentos discretos com a progressão da idade, como o de Leboeuf-Yde et al. 18 realizado na Dinamarca e que, no entanto, verificaram dores com duração mais prolongada entre os mais idosos, achado concordante com os resultados do estudo de Silva et al. ${ }^{8}$ desenvolvido em Pelotas, em 2002.
Dados da PNAD apontaram aumento progressivo da prevalência desde a infância até a faixa de 60 a 69 anos, quando o coeficiente estabilizou e sofreu apenas pequeno declínio na faixa de 80 anos e mais 2 . A maior prevalência das dores entre os idosos decorreria do efeito cumulativo de exposições a esforços físicos e lesões durante a vida, concomitantemente às modificações anatômicas na estrutura espinal que ocorrem com o envelhecimento 15 . 
Tabela 3

Prevalência de dor nas costas segundo morbidades e problemas de saúde na população adulta (18 a 59 anos) de Campinas, São Paulo, Brasil, em 2008. Inquérito de Saúde de Campinas (ISACamp), 2008/2009.

\begin{tabular}{|c|c|c|c|c|}
\hline Morbidades e problemas de saúde & $\mathrm{n}$ & Prevalência (\%) & RP bruta (IC95\%) & RP ajustada * (IC95\%) \\
\hline Hipertensão & & $p<0,0001$ ** & & \\
\hline Sim & 142 & 48,7 & $1,76(1,42-2,18)$ & $1,38(1,10-1,72)$ \\
\hline Não & 976 & 27,7 & 1,00 & \\
\hline Diabetes & & $p=0,2708$ ** & & \\
\hline Sim & 37 & 37,7 & $1,24(0,86-1,80)$ & $0,92(0,63-1,35)$ \\
\hline Não & 1.077 & 30,4 & 1,00 & 1,00 \\
\hline Artrite/Reumatismo/Artrose & & $p<0,0001 * \star$ & & \\
\hline Sim & 37 & 60,1 & $2,03(1,57-2,63)$ & $1,41(1,05-1,90)$ \\
\hline Não & 1.080 & 29,6 & 1,00 & 1,00 \\
\hline Número de doenças crônicas & & $p<0,0001 * *$ & & \\
\hline Nenhuma & 714 & 25,2 & 1,00 & 1,00 \\
\hline 1 & 267 & 34,6 & $1,37(1,10-1,71)$ & $1,22(0,97-1,53)$ \\
\hline 2 & 76 & 42,7 & $1,69(1,21-2,38)$ & $1,36(0,95-1,94)$ \\
\hline 3 ou mais & 51 & 61,2 & $2,43(1,81-3,25)$ & $1,72(1,24-2,39)$ \\
\hline Dor de cabeça/Enxaqueca & & $\mathrm{p}<0,0001 \star \star \star$ & & \\
\hline Sim & 317 & 47,7 & $1,98(1,67-2,36)$ & $1,96(1,65-2,33)$ \\
\hline Não & 801 & 24,0 & 1,00 & 1,00 \\
\hline Tendinite/LER/DORT & & $p=0,0331 * *$ & & \\
\hline Sim & 60 & 42,7 & $1,43(1,05-1,96)$ & $1,26(0,94-1,68)$ \\
\hline Não & 1.056 & 29,8 & 1,00 & 1,00 \\
\hline Insônia & & $p<0,0001 * *$ & & \\
\hline Sim & 159 & 50,5 & $1,86(1,50-2,30)$ & $1,56(1,25-1,95)$ \\
\hline Não & 959 & 27,2 & 1,00 & 1,00 \\
\hline Número de problemas de saúde *** & & $p<0,0001 * *$ & & \\
\hline Nenhum & 404 & 15,5 & 1,00 & \\
\hline 1 & 352 & 34,2 & $2,21(1,58-3,08)$ & $2,10(1,49-2,96)$ \\
\hline 2 & 191 & 36,3 & $2,34(1,60-3,40)$ & $2,20(1,52-3,18)$ \\
\hline 3 ou mais & 171 & 52,7 & $2,39(2,51-4,60)$ & $2,97(2,14-4,11)$ \\
\hline Transtorno mental comum & & $p=0,0002 \star \star$ & & \\
\hline Sim & 109 & 49,2 & $1,72(1,35-2,18)$ & $1,49(1,16-1,92)$ \\
\hline Não & 1.008 & 28,6 & 1,00 & 1,00 \\
\hline IMC & & $p=0,0001 \star \star *$ & & \\
\hline Eutrófico & 618 & 25,2 & 1,00 & 1,00 \\
\hline Sobrepeso & 324 & 33,8 & $1,34(1,07-1,68)$ & 1,23 (0,99-1,53) \\
\hline Obeso & 176 & 41,8 & $1,66(1,33-2,07)$ & $1,43(1,13-1,80)$ \\
\hline Autoavaliação da saúde & & $p<0,0001 * *$ & & \\
\hline Excelente & 216 & 15,0 & 1,00 & 1,00 \\
\hline Muito boa & 271 & 23,2 & $1,55(0,91-2,64)$ & $1,50(0,86-2,62)$ \\
\hline Boa & 565 & 37,4 & $2,49(1,63-3,81)$ & $2,29(1,46-3,59)$ \\
\hline Ruim/Muito ruim & 66 & 52,6 & $3,51(2,08-5,90)$ & $2,93(1,69-5,07)$ \\
\hline
\end{tabular}

IC95\%: intervalo de 95\% de confiança; IMC: índice de massa corporal; RP: razão de prevalência.

* Ajustada por idade e sexo;

** Valor de $p$ do teste qui-quadrado;

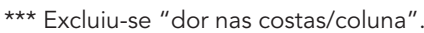


Tabela 4

Modelos de regressão múltipla de Poisson. Inquérito de Saúde de Campinas (ISACamp), 2008/2009.

\begin{tabular}{|c|c|c|c|c|}
\hline Variáveis & $\begin{array}{c}\text { Primeira etapa } \\
\text { RP ajustada * (IC95\%) }\end{array}$ & $\begin{array}{c}\text { Segunda etapa } \\
\text { RP ajustada ** (IC95\%) }\end{array}$ & $\begin{array}{c}\text { Terceira etapa } \\
\text { RP ajustada *** (IC95\%) }\end{array}$ & $\begin{array}{c}\text { Quarta etapa } \\
\text { RP ajustada \# (IC95\%) }\end{array}$ \\
\hline \multicolumn{5}{|l|}{ Sexo } \\
\hline Masculino & 1,00 & 1,00 & 1,00 & 1,00 \\
\hline Feminino & $1,24(1,00-1,54)$ & $1,25(1,02-1,53)$ & $1,07(0,88-1,30)$ & $1,06(0,88-1,28)$ \\
\hline \multicolumn{5}{|l|}{ Faixa etária (anos) } \\
\hline $18-29$ & 1,00 & 1,00 & 1,00 & 1,00 \\
\hline $30-39$ & $1,20(0,87-1,65)$ & $1,22(0,90-1,65)$ & $1,21(0,89-1,65)$ & $1,16(0,87-1,55)$ \\
\hline $40-49$ & $1,19(0,86-1,63)$ & $1,19(0,88-1,59)$ & $1,14(0,85-1,52)$ & $1,06(0,80-1,40)$ \\
\hline $50-59$ & $1,35(1,00-1,83)$ & $1,37(1,03-1,82)$ & $1,27(0,95-1,70)$ & $1,20(0,91-1,59)$ \\
\hline \multicolumn{5}{|l|}{ Número de filhos } \\
\hline 0 & 1,00 & 1,00 & 1,00 & 1,00 \\
\hline 1 a 2 & $1,42(1,05-1,92)$ & $1,26(0,93-1,72)$ & $1,27(0,93-1,72)$ & $1,31(1,97-1,56)$ \\
\hline 3 ou mais & $2,23(1,54-3,22)$ & $1,80(1,24-2,63)$ & $1,70(1,20-2,41)$ & $1,67(1,20-2,33)$ \\
\hline \multicolumn{5}{|l|}{ Carga horária semanal de } \\
\hline \multicolumn{5}{|l|}{ trabalho (horas) } \\
\hline Não trabalha & 1,00 & 1,00 & 1,00 & 1,00 \\
\hline$<40$ & $1,14(0,86-1,50)$ & $1,15(0,83-1,59)$ & $1,12(0,80-1,56)$ & $1,04(0,74-1,45)$ \\
\hline 40 & $1,09(0,77-1,53)$ & $1,05(0,76-1,45)$ & $1,06(0,76-1,48)$ & $1,02(0,73-1,43)$ \\
\hline$>40$ & $1,36(1,00-1,85)$ & $1,24(0,94-1,65)$ & $1,23(0,93-1,63)$ & $1,20(0,91-1,58)$ \\
\hline \multicolumn{5}{|l|}{ Tabagismo } \\
\hline Nunca fumou & & 1,00 & 1,00 & 1,00 \\
\hline Fumante & & $1,25(0,99-1,57)$ & $1,20(0,95-1,51)$ & $1,18(0,93-1,49)$ \\
\hline Ex-fumante & & $1,48(1,13-1,93)$ & $1,36(1,05-1,75)$ & $1,33(1,02-1,72)$ \\
\hline \multicolumn{5}{|l|}{ Atividade física no lazer } \\
\hline Ativo & & 1,00 & 1,00 & 1,00 \\
\hline Insuficientemente ativo & & $1,24(0,80-1,92)$ & $1,27(0,83-1,95)$ & $1,20(0,80-1,80)$ \\
\hline Inativo & & $1,40(1,06-1,83)$ & $1,35(1,02-1,78)$ & $1,24(0,95-1,63)$ \\
\hline \multicolumn{5}{|l|}{ Atividade física doméstica } \\
\hline Ativo & & 1,00 & 1,00 & 1,00 \\
\hline Insuficientemente ativo & & $0,70(0,51-0,97)$ & $0,71(0,51-1,03)$ & $0,77(0,54-1,09)$ \\
\hline Inativo & & $0,78(0,61-0,99)$ & $0,78(0,62-0,99)$ & $0,79(0,63-0,99)$ \\
\hline \multicolumn{5}{|l|}{ Atividade física no trabalho } \\
\hline Ativo & & 1,00 & 1,00 & 1,00 \\
\hline Ativo & & $1,37(0,96-1,94)$ & $1,39(0,97-1,98)$ & $1,43(0,98-2,08)$ \\
\hline Insuficientemente ativo & & $0,72(0,56-0,94)$ & $0,76(0,58-0,99)$ & $0,79(0,61-1,02)$ \\
\hline \multicolumn{5}{|l|}{ Horas assistindo televisão } \\
\hline Até 1 & & 1,00 & 1,00 & 1,00 \\
\hline 2 & & $0,91(0,70-1,18)$ & $0,90(0,68-1,18)$ & $0,91(0,70-1,18)$ \\
\hline 3 & & $1,12(0,87-1,44)$ & $1,07(0,85-1,34)$ & $1,05(0,83-1,32)$ \\
\hline 4 ou mais & & $1,35(1,02-1,78)$ & $1,19(0,93-1,52)$ & $1,18(0,93-1,51)$ \\
\hline \multicolumn{5}{|c|}{ Número de problemas de saúde } \\
\hline 0 & & & 1,00 & 1,00 \\
\hline 1 & & & $1,94(1,41-2,67)$ & $1,94(1,40-2,68)$ \\
\hline 2 & & & $2,05(1,41-2,98)$ & $1,96(1,35-2,86)$ \\
\hline 3 ou mais & & & $2,65(1,87-3,74)$ & $2,42(1,73-3,39)$ \\
\hline \multicolumn{5}{|l|}{ Autoavaliação da saúde } \\
\hline Excelente & & & & 1,00 \\
\hline Muito boa & & & & $1,59(0,97-2,61)$ \\
\hline Boa & & & & $1,92(1,30-2,85)$ \\
\hline Ruim/Muito ruim & & & & $2,08(1,26-3,44)$ \\
\hline
\end{tabular}

IC95\%: intervalo de 95\% de confiança; RP: razão de prevalência.

* Ajustada pelas variáveis demográficas e socioeconômicas;

** Ajustada pelas variáveis da primeira etapa e pelas variáveis de comportamentos relacionados à saúde;

***Ajustada pelas variáveis da primeira e segunda etapas e pelas variáveis de condições de saúde;

\# Ajustada por todas as variáveis do modelo e autoavaliação da saúde. 
No presente trabalho, a associação das dores nas costas com o número de filhos persistiu mesmo após o ajuste para todas as variáveis do modelo, e a associação está presente nos homens e nas mulheres. Analisando a associação entre número de filhos e dores nas costas, Silman et al. 25 encontraram um risco aumentado para casados de ambos os sexos comparados com os solteiros e, entre os casados, um crescente aumento de risco proporcional ao aumento do número de filhos. Entretanto, em estudo específico com mulheres não foi encontrada associação positiva de lombalgia com número de filhos 19. O número de filhos até o momento não foi avaliado em relação à presença de dor nas costas em estudos brasileiros. A associação é pouco estudada e novas pesquisas devem ser desenvolvidas para verificar a consistência e o entendimento da associação.

A escolaridade não persistiu associada à dor nas costas no modelo final; somente a carga horária de trabalho semanal superior a 40 horas revelou associação positiva com dores nas costas no limiar de significância estatística. Entretanto, outros estudos brasileiros 2,8 mostraram prevalência de doenças da coluna/das costas significativamente maior entre os de menor escolaridade. Bassols et al. ${ }^{5}$ encontraram maior prevalência de dores lombares em indivíduos com baixa escolaridade e nos que realizavam trabalhos braçais 5 . Um estudo de revisão indicou que nível de escolaridade tem impacto na duração e recorrência dos episódios de dor e os de maior escolaridade apresentam evolução mais favorável do quadro álgico. Esse fator pode estar associado com o status socioeconômico, tipo de ocupação, hábitos $\mathrm{e}$ fatores ambientais 15 .

A associação de dores nas costas com o consumo de tabaco tem sido observada na maior parte das pesquisas que analisaram esta questão, sendo em geral de baixa magnitude 15,26,27. Associação forte foi observada para lombalgia crônica e incapacidade, e ex-fumantes apresentaram prevalências menores do que os fumantes ativos 26. Em um estudo desenvolvido na Dinamarca encontrou-se forte associação entre o consumo do tabaco e dores lombares, com nítido gradiente dose-resposta 28 . Em outro trabalho realizado no mesmo país, constatou-se associação entre a prevalência de dores lombares e o consumo "pesado" de tabaco 29. Essa associação também foi observada na Holanda 22, mas em estudo conduzido na Turquia ela não foi registrada ${ }^{30}$. No Brasil, a associação de dor nas costas com o tabagismo foi encontrada nas populações de Pelotas 5,8 e Salvador 7 . Várias hipóteses têm sido aventadas para explicar a maior prevalência de dor lombar nos fumantes, como o aumento da pressão intraabdominal devido à tosse crônica, as alterações hormonais, a redução do fluxo sanguíneo na região da coluna, a osteopenia com microfraturas trabeculares na coluna e os defeitos fibrinolíticos causando alterações discais 15; a nicotina também poderia reduzir a perfusão em discos intervertebrais, aumentando os níveis de citocinas pró-inflamatórias e potencializando a transmissão de sinais álgicos ao sistema nervoso central 7 . Entre os fumantes pesados também foram encontradas prevalências mais elevadas de "processo inflamatório vertebral" (vertebral inflammatory process - VIP) associadas a quadros álgicos 26 .

A associação entre dores nas costas e atividade física em contexto de lazer detectada na população de Campinas também foi constatada em estudos transversais realizados em Israel 31 e na Suécia 32. Em um estudo realizado na Noruega, estimou-se que havia risco aumentado de desenvolver quadro de dores crônicas em indivíduos fisicamente inativos 33. Entretanto, em revisão sobre dor nas costas e atividade física que incluiu 13 estudos, sendo dez transversais e três longitudinais, os autores concluíram que, baseados no pequeno número de estudos e na heterogeneidade entre eles, havia limitada evidência de associação entre atividade física no tempo de lazer e lombalgias ou cervicalgias, concluindo pela necessidade de novos trabalhos para melhor elucidar esta questão 34 . Em outro estudo também foram encontradas evidências limitadas para sustentar a hipótese de que trabalhos sedentários ou permanecer sentado durante o trabalho por períodos prolongados sejam fatores de risco para lombalgias, assim como para comportamentos sedentários/permanecer sentado por períodos prolongados 35 .

No presente estudo, os que realizavam atividade doméstica e de trabalho apresentaram maiores prevalências de dor nas costas. Em estudo caso-controle australiano, com 486 gêmeos, a lombalgia foi significativamente associada ao trabalho doméstico pesado ( $\mathrm{OR}=2,88$, IC95\%: 1,29-6,43) e não associada a qualquer forma de atividade recreacional 36 . Em inquérito populacional na Suécia, os que apresentaram lombalgia realizavam mais frequentemente trabalho físico pesado e menor atividade física no lazer, além de estarem entre os que fumavam, os com maior IMC e com menor escolaridade 32 .

Autores têm descrito a redução de atividade provocada pela presença da algia lombar. Leboeuf-Yde et al. 18 observaram que uma das consequências das dores nas costas para a população estudada era a redução das atividades físicas, dificultando assim considerar se seria um 
fator para o acometimento ou um resultante do processo álgico. Em outro estudo, descreveu-se a restrição das atividades físicas em cerca de metade dos lombálgicos 37 .

A associação de lombalgia com obesidade verificada na população de Campinas $(\mathrm{RP}=1,39)$, na análise ajustada apenas por idade e sexo, também tem sido relatada em outros estudos. Uma pesquisa conduzida na Noruega constatou que homens e mulheres obesos apresentaram aumento do risco de dores crônicas nas costas 32 e a obesidade é considerada um preditor destas dores e de sua gravidade 15. Uma meta-análise realizada indica que sobrepeso e obesidade aumentam o risco de lombalgias, e apresenta forte associação com busca de cuidados à saúde por lombalgia e lombalgia crônica 38 . Mas, no presente estudo, a variável IMC, quando incluída na terceira etapa do modelo, e sofrendo, portanto, ajuste pelas variáveis que persistiram nas primeiras e segundas etapas e da variável incluída na terceira etapa (número de doenças crônicas), perdeu significância estatística não tendo permanecido no modelo final.

Aqueles que relataram assistir à televisão por quatro horas ou mais no dia apresentaram probabilidade $35 \%$ maior de ter dor nas costas; entretanto, não encontramos estudos avaliando este fator. As pesquisas que analisam o tempo de assistir à televisão estão relacionadas em geral ao comportamento sedentário e à sua associação com sobrepeso/obesidade e outras morbidades. Ressalte-se que em nosso estudo a associação da algia nas costas com o tempo de assistir à televisão perde significância na terceira etapa do modelo quando a variável número de problemas de saúde foi introduzida. Assim, pesquisas devem ser desenvolvidas para verificar a consistência e o entendimento da associação.

Na população estudada, indivíduos com outras morbidades e problemas de saúde como hipertensão, artrite/reumatismo, artrose, dor de cabeça/enxaqueca, insônia e transtorno mental comum apresentaram prevalências mais elevadas de dor nas costas. No modelo final permaneceu com associação significativa a variável sintética "número de problemas de saúde". Uma pesquisa longitudinal com gêmeos desenvolvida na Dinamarca entre os anos de 1995 e 2001 revelou que dores nas costas estavam associadas à extensa lista de comorbidades, tanto em análise univariada como na análise multivariada. As mais destacadas foram as doenças musculoesqueléticas, entretanto, cefaleia, doenças respiratórias, cardiovasculares e a úlcera gástrica também foram significantemente associadas às dores nas costas 39 . Comparados com a população normal de referência, pacientes com lombalgia apresentaram mais dorsalgia, cervicalgia, dores em membros inferiores ao realizar exercícios, cefaleia, enxaqueca, problemas para dormir, ondas de calor, ansiedade, tristeza e depressão em pesquisa conduzida na Noruega 40. Os estudos brasileiros de base populacional já publicados sobre o tema não haviam avaliado a associação entre dor nas costas e outras morbidades.

Adultos que avaliaram a própria saúde como boa e ruim ou muito ruim apresentaram maior prevalência de dor nas costas, chegando até o dobro da frequência entre os que relataram saúde ruim ou muito ruim em relação aos que a avaliaram como excelente (RP ajustada $=2,02$ ). Piores autoavaliações do estado geral de saúde foram também associadas positivamente com lombalgia em estudos conduzidos em Israel $31 \mathrm{e} \mathrm{em}$ Pelotas 9 . As associações encontradas entre lombalgias e comorbidades explicariam em parte a associação entre pior autoavaliação de saúde e lombalgias, mas a associação, no presente trabalho, persiste no modelo final mesmo após ajuste para o número de problemas de saúde.

Dado o corte transversal do presente estudo, as associações encontradas não podem ser consideradas de causa-efeito. Não é possível, por exemplo, saber se a falta de atividade física em lazer teria aumentado o risco de lombalgia ou se a presença de lombalgia reduziu a prática de atividade física. Por não se tratar de um estudo especifico para as algias de coluna, não há informações sobre a localização da dor e detalhamento dos eventos.

As comparações dos achados com dados de outras pesquisas requerem considerar as diferenças existentes quanto aos métodos empregados, o período de tempo da dor referida, a localização da dor, o tipo e tamanho da amostra, a faixa de idade considerada, entre outros. Os trabalhos publicados apresentam grande variação na prevalência de lombalgias, dorsalgias e cervicalgias, atribuídas às variações nos métodos de pesquisa e na forma de conceituar os sintomas apresentados, além das diferenças "verdadeiras" entre as populações e segmentos estudados.

Neste estudo, a prevalência de dor nas costas/ problema na coluna foi elevada, aumentando com a idade e significativamente mais alta entre as mulheres. Houve maior prevalência de dor entre os indivíduos com a carga horária de trabalho semanal superior a 40 horas, nos fumantes, nos inativos durante o lazer e ativos em atividades domésticas e de trabalho, sendo a prevalência positivamente associada ao número de filhos.

Os inquéritos populacionais gerais de saúde, mesmo sem detalhamento específico para dores nas costas, permitem descrever as prevalências gerais e suas principais características. Com 
base em inquérito geral de saúde, este trabalho possibilitou estimar a prevalência do problema e identificar os segmentos demográficos e sociais com as maiores prevalências de dores nas costas a receber atenção dos profissionais e dos serviços de saúde envolvidos.

\section{Resumen}

Este estudio buscó estimar la prevalencia de dolor de espalda en la población urbana de Campinas, São Paulo, Brasil, y también identificar los subgrupos de la población más afectados por el problema. Los datos se obtuvieron a partir de la encuesta de población realizada entre los años 2008 y 2009 y que involucró 1.118 personas con edades entre 18 a 59 años. La prevalencia del dolor de espalda fue de $30,6 \%$, afectando $34,4 \%$ de mujeres y 26,5\% de hombres. Después del ajuste, la prevalencia se mostró más elevada entre las mujeres, las personas con menor número de años de estudio, entre trabajadores con más de 40 horas a la semana, en los fumadores y ex fumadores, en los quienes no practicaban actividad fisica en su tiempo libre y en quienes realizaban actividades domésticas y de trabajo. La prevalencia aumentó con la edad y con el número de hijos, con el número de morbilidades y problemas de salud y con la peor autopercepción de salud. Los resultados confirman la alta prevalencia del problema y las limitaciones que causa, indicando los segmentos demográficos que merecen más atención por parte de los servicios de salud.

Dolor de Espalda; Salud del Adulto;

Encuestas Epidemiológicas
Inquéritos específicos são sugeridos para permitir maior conhecimento sobre este problema altamente prevalente na população.

\section{Colaboradores}

A. M. Iguti foi redatora principal do texto, realizou a revisão bibliográfica e participou da análise e discussão de dados. T. F. Bastos participou no desenvolvimento das análises estatísticas e na revisão do texto. M. B. A. Barros colaborou no delineamento e na coleta de dados do estudo, na análise estatística, bem como na revisão final do texto.

\section{Agradecimentos}

Ao CNPq (processo no 409747/2006-8) pelo financiamento da pesquisa e pela bolsa de produtividade de $\mathrm{M}$. B. A. Barros; à FAPESP pela bolsa de doutorado de T. F. Bastos (processo no 2012/07970-2) e ao Ministério da Saúde e à Secretaria de Saúde de Campinas pelo apoio financeiro para a realização do inquérito (Convênio Unicamp/Funcamp/SMS no 4300). 


\section{Referências}

1. Hoy D, Bain C, Williams G, March L, Brooks P Blyth F, et al. A systematic review of the global prevalence of low back pain. Arthritis Rheum 2012; 64:2028-37.

2. Barros MBA, Francisco PMSB, Zanchetta LM, César CLG. Tendências das desigualdades sociais e demográficas na prevalência de doenças crônicas no Brasil, PNAD: 2003-2008. Ciênc Saúde Coletiva 2011; 16:3755-68.

3. Hoy D, Brook P, Blythc F, Buchbinder R. The epidemiology of low back pain. Best Pract Res Clin Rheumatol 2010; 24:769-81.

4. Picavet HS, Struijs JN, Westert GP. Utilization of health resources due to low back pain: survey and registered data compared. Spine (Phila Pa 1976) 2008; 33:43-4.

5. Bassols A, Boscha F, Campillob M, Bañosa JE. El dolor de espalda en la población catalana. Prevalencia, características y conducta terapéutica. Gac Sanit 2003; 17:97-107.

6. Hoy D, March L, Brooks P, Blyth F, Woolf A, Bain $C$, et al. The global burden of low back pain: estimates from the Global Burden of Disease 2010 study. Ann Rheum Dis 2014; 73:968-74.

7. Meucci RD, Fassa AG, Paniz VM, Silva MC, Wegman DH. Increase of chronic low back pain prevalence in a medium-sized city of southern Brazil. BMC Musculoskelet Disord 2013; 14:155.

8. Silva MC, Fassa AG, Valle NCJ. Dor lombar crônica em uma população adulta do Sul do Brasil: prevalência e fatores associados. Cad Saúde Pública 2004; 20:377-85.

9. Ferreira GD, Silva MC, Rombaldi AJ, Wrege ED, Siqueira FV, Hallal PC. Prevalência de dor nas costas e fatores associados em adultos do Sul do Brasil: estudo de base populacional. Rev Bras Fisioter 2011; 15:31-6.

10. Almeida ICGB, Sá KN, Silva M, Baptista A, Matos MA, Lessa I. Prevalência de dor lombar crônica na população da Cidade de Salvador. Rev Bras Ortop 2008; 43:96-102.

11. Alves MCGP. ISA-Campinas 2008-2009: plano de amostragem. http://www.fcm.unicamp.br/fcm/ sites/default/files/plano_de_amostragem.pdf (acessado em 02/Mar/2015)

12. Babor TF, Higgins-Biddle JC, Saunders JB, Monteiro MG. AUDIT: The alcohol use disorders identification test: guidelines for use in primary health care. 2nd Ed. Geneva: World Health Organization; 2001.

13. World Health Organization. Obesity: preventing and managing the global epidemic. Geneva: World Health Organization; 2000. (WHO Technical Report Series, 894).

14. Mari JJ, Willians P. A validity study of a Psychiatric Screening Questionnaire (SRQ-20) in primary care in the city of São Paulo. Br J Psychiatry 1986; 148:23-6.
15. Rubin DI. Epidemiology and risk factors for spine pain. Neurol Clin 2007; 25:353-71.

16. Salaffi F, De Angelis R, Grassi W. Prevalence of musculoskeletal conditions in an Italian population sample: results of a regional community-based study. I. The MAPPING study. Clin Exp Rheumatol 2005; 23:819-28.

17. Kopec JA, Sayre EC, Esdaile JM. Predictors of back pain in a general population cohort. Spine (Phila Pa 1976) 2004; 29:70-7.

18. Leboeuf-Yde C, Nielsen J, Kyvik KO, Fejer R, Hartvigsen J. Pain in the lumbar, thoracic or cervical regions: do age and gender matter? A populationbased study of 34,902 Danish twins 20-71 years of age. BMC Musculoskelet Disord 2009; 10:39.

19. Wijnhoven HA, de Vet HC, Smit HA, Picavet HS. Hormonal and reproductive factors are associated with chronic low back pain and chronic upper extremity pain in women: the MORGEN study. Spine (Phila Pa 1976) 2006; 31:1496-502.

20. Wijnhoven HA, de Vet HC, Picavet HS. Sex differences in consequences of musculoskeletal pain. Spine (Phila Pa 1976) 2007; 32:1360-7.

21. Plouvier S, Gourmelen J, Chastang JF, Lanoë JL, Leclerc A. Low back pain around retirement age and physical occupational exposure during working life. BMC Public Health 2011; 11:268.

22. van Oostrom SH, Monique Verschuren WM, de Vet HC, Picavet HS. Ten year course of low back pain in an adult population-based cohort: the Doetinchem cohort study. Eur J Pain 2011; 15:993-8.

23. Niemeläinen R, Videman T, Battié MC. Prevalence and characteristics of upper or mid-back pain in Finnish men. Spine (Phila Pa 1976) 2006; 31:18469.

24. Webb R, Brammah T, Lunt M, Urwin M, Allison T, Symmons D. Prevalence and predictors of intense, chronic, and disabling neck and back pain in the UK general population. Spine (Phila Pa 1976) 2003; 28:1195-202.

25. Silman AJ, Ferry S, Papageorgiou AC, Jayson MI, Croft PR. Number of children as a risk factor for low back pain in men and women. Arthritis Rheum 1995; 38:1232-5.

26. Leboeuf-Yde C, Kjær P, Bendix T, Manniche C. Selfreported hard physical work combined with heavy smoking or overweight may result in so-called Modic changes. BMC Musculoskelet Disord 2008; 9:5.

27. Shiri R, Karppinen J, Leino-Arjas P, Solovieva S, Viikari-Juntura E. The association between smoking and low back pain: a meta-analysis. Am J Med 2010; 123:87.e7-35.

28. Pisinger C, Aadahl M, Toft U, Birke H, ZytphenAdeler J, Jørgensen T. The association between active and passive smoking and frequent pain in a general population. Eur J Pain 2011; 15:77-83. 
29. Jensen TS, Kjaer P, Korsholm L, Bendix T, Sorensen JS, Manniche C, et al. Predictors of new vertebral endplate signal (Modic) changes in the general population. Eur Spine J 2010; 19:129-35.

30. Oksuz E. Prevalence, risk factors, and preferencebased health states of low back pain in a Turkish population. Spine 2006; 31:968-72.

31. Jacob T. Low back pain incident episodes: a community-based study. Spine J 2006; 6:306-10.

32. Nilsen TI, Holtermann A, Mork PJ. Physical exercise, body mass index, and risk of chronic pain in the low back and neck/shoulders: longitudinal data from the Nord-Trondelag Health Study. Am J Epidemiol 2011; 174:267-73.

33. Björck-van Dijken C, Fjellman-Wiklund A, Hildingsson C. Low back pain, lifestyle factors and physical activity: a population-based study. J Rehabil Med 2008; 40:864-9.

34. Sitthipornvorakul E, Janwantanakul P, Purepong N, Pensri P, van der Beek AJ. The association between physical activity and neck and low back pain: a systematic review. Eur Spine J 2010; 20: 677-89.

35. Chen S-M, Liu M-F, Cook J, Shona Bass S, Lo SK. Sedentary lifestyle as a risk factor for low back pain: a systematic review. Int Arch Occup Environ Health 2009; 82:797-806.
36. Hübscher M, Ferreira ML, Junqueira DRG, Refshauge KM, Maher CG, Hopper JL, et al. Heavy domestic, but not recreational, physical activity is associated with low back pain: Australian Twin low BACK pain (AUTBACK) study. Eur Spine J 2014; 23:2083-9.

37. McKinnon ME, Vickers MR, Ruddock VM, Townsend J, Meade TW. Community studies of the health service implications of low back pain. Spine (Phila Pa 1976) 1997; 22:2161-6.

38. Shiri R, Karppinen J, Leino-Arjas P, Solovieva S, Viikari-Juntura E. The association between obesity and low back pain: a meta-analysis. Am J Epidemiol 2010; 171:135-54.

39. Hartvigsen J, Christensen K, Frederiksen H. Back pain remains a common symptom in old age. A population-based study of 4486 Danish twins aged 70-102. Eur Spine J 2003; 12:528-34.

40. Hagen EM, Svensen E, Eriksen HR, Ihlebaek CM, Ursin H. Comorbid subjective health complaints in low back pain. Spine (Phila Pa 1976) 2006; 31:1491-5.

Recebido em 28/Nov/2014

Versão final reapresentada em 18/Mai/2015

Aprovado em 08/Jun/2015 